SECTION 2. Applied mathematics. Mathematical modeling.

Naumov Anatoly Aleksandrovich Docent, candidate of Technical Sciences, Center of Applied Mathematical Research, Novosibirsk, Russia, E-mail: A_A_Naumov@mail.ru

\title{
TO S_P-INVARIANCE PROPERTY OF MULTICRITERIA OPTIMIZATION PROBLEMS METHODS
}

In the paper the invariance of the method of normalization criteria used to solve multi-criteria optimization problems is studied.

Keywords: multi-criteria problems, method of criteria normalization, invariance.

\section{К СВОЙСТВУ $\boldsymbol{S}_{\boldsymbol{p}}$-ИНВАРИАНТНОСТИ МЕТОДОВ РЕШЕНИЯ МНОГОКРИТЕРИАЛЬНЫХ ЗАДАЧ ОПТИМИЗАЦИИ}

В работе исследован на инвариантность метод нормализаџии критериев, используемыгй для решения многокритериальных задач оптимизачии.

Ключевые слова: многокритериальные задачи, метод нормализачии критериев, инвариантность.

В работе рассмотрено свойство инвариантности относительно множества пассивных ограничений метода нормализации критериев (см. [1]-[4]) решения многокритериальных задач оптимизации.

Постановка задачи и основные определения. Введем обозначения для задачи многокритериальной оптимизации. Целевые функции задачи сведем в единый вектор -

$$
\mathrm{F}=\left(\mathrm{f}_{1}\left(\mathrm{x}_{1}, \mathrm{x}_{2}, \mathrm{x}_{3}, \ldots, \mathrm{x}_{\mathrm{n}}\right), \mathrm{f}_{2}\left(\mathrm{x}_{1}, \mathrm{x}_{2}, \mathrm{x}_{3}, \ldots, \mathrm{x}_{\mathrm{n}}\right), \ldots, \mathrm{f}_{\mathrm{p}}\left(\mathrm{x}_{1}, \mathrm{x}_{2}, \mathrm{x}_{3}, \ldots, \mathrm{x}_{\mathrm{n}}\right)\right)
$$

и будем считать, что все функции этого вектора необходимо максимизировать. Ограничения задачи объединим во множество $\mathrm{S}=$ $\left\{g_{1}\left(x_{1}, x_{2}, x_{3}, \ldots, x_{n}\right) \leq b_{1}, g_{2}\left(x_{1}, x_{2}, x_{3}, \ldots, x_{n}\right) \leq\right.$ $\left.\mathrm{b}_{2}, \ldots, \mathrm{g}_{\mathrm{m}}\left(\mathrm{x}_{1}, \mathrm{x}_{2}, \mathrm{x}_{3}, \ldots, \mathrm{x}_{\mathrm{n}}\right) \leq \mathrm{b}_{\mathrm{m}}\right\}$.

Тогда, задачу многокритериальной оптимизации обозначим в виде кортежа: $\mathrm{P}=\langle\mathrm{F}, \mathrm{S}\rangle$. Последняя запись читается таким образом: требуется найти максимум векторной функции $F$ при ограничениях S. Введем в рассмотрение определения. Парето-множество задачи $\mathrm{P}=\langle\mathrm{F}, \mathrm{S}\rangle$ обозначим через $\mathrm{D}_{\pi}$, а множество ограничений задачи его определяющее через $S_{\pi}$ и назовем множеством активных ограничений. Множество 
ограничений из $S$, не входящее во множество активных ограничений $S_{\pi}$, назовем множеством пассивных ограничений задачи $\mathrm{P}=\langle\mathrm{F}, \mathrm{S}\rangle$ и обозначим через $S_{p}$. Таким образом, $S=S_{\pi} \cup S_{p}$, а $S_{\pi} \cap S_{p}=\emptyset$. Метод (M) решения задачи $\langle F, S\rangle$ - это отображение пары $\langle F, S\rangle$ в некоторую область (точку) $\mathrm{x}^{*}$ области $\mathrm{D}_{\pi}$, т.е. $\langle\mathrm{F}, \mathrm{S}\rangle \stackrel{\mathrm{M}}{\rightarrow} \mathrm{x}^{*} \in \mathrm{D}_{\pi} \quad$ или $\mathrm{M}:\langle\mathrm{F}, \mathrm{S}\rangle \rightarrow \mathrm{x}^{*} \in \mathrm{D}_{\pi}$ (для точки $\left.x^{*}\right)$. Метод (М) решения задачи $(\mathrm{P}=\langle\mathrm{F}, \mathrm{S}\rangle)$ является $\boldsymbol{S}_{\boldsymbol{p}^{-}}$ инвариантным, если решение задачи, найденное этим методом, не меняется при изменении элементов множества $S_{p}$. При этом считается, что элементы вектора $\mathrm{F}$ и множества $\mathrm{D}_{\pi}$ являются фиксированными и не изменяются при изменении $S_{p}$.

Пример. Рассмотрим две задачи линейного программирования.

Задача А. Пусть задан вектор критериев $\mathrm{F}_{\mathrm{A}}=\left(\mathrm{f}_{1, \mathrm{~A}}\left(\mathrm{x}_{1}, \mathrm{x}_{2}\right), \mathrm{f}_{2, \mathrm{~A}}\left(\mathrm{x}_{1}, \mathrm{x}_{2}\right)\right)=\left(1000 \cdot \mathrm{x}_{2}, 0.1 \cdot \mathrm{x}_{1}\right) \quad$ и ограничения $\mathrm{S}_{\mathrm{A}}=\left\{\mathrm{g}_{1, \mathrm{~A}}\left(\mathrm{x}_{1}, \mathrm{x}_{2}\right) \equiv \mathrm{x}_{1}+\mathrm{x}_{2} \leq 2, \mathrm{~g}_{2, \mathrm{~A}}\left(\mathrm{x}_{1}, \mathrm{x}_{2}\right) \equiv \mathrm{x}_{1}-\mathrm{x}_{2} \geq-1, \mathrm{~g}_{3, \mathrm{~A}}\left(\mathrm{x}_{1}, \mathrm{x}_{2}\right) \equiv\right.$ $\left.\mathrm{x}_{1}-\mathrm{x}_{2} \leq 1, \mathrm{~g}_{4, \mathrm{~A}}\left(\mathrm{x}_{1}, \mathrm{x}_{2}\right) \equiv \mathrm{x}_{1} \geq 0, \mathrm{~g}_{5, \mathrm{~A}}\left(\mathrm{x}_{1}, \mathrm{x}_{2}\right) \equiv \mathrm{x}_{2} \geq 0\right\}$. Необходимо найти решение этой задачи: $\mathrm{F}_{\mathrm{A}} \rightarrow \max$ при ограничениях $\mathrm{S}_{\mathrm{A}}$.

Решаем задачу методом нормализации критериев (см. [1]-[4]). Последовательно находим: $\mathrm{f}_{1, \mathrm{~A}}^{\max }=1500$ (достигается в точке $(0.5,1.5)$; $\mathrm{f}_{1, \mathrm{~A}}^{\min }=0$ (достигается в на отрезке $([0 ; 1], 0) ; \mathrm{f}_{2, \mathrm{~A}}^{\max }=0.15$ (достигается в точке $(1.5,0.5) ; \mathrm{f}_{2, A}^{\min }=0$ (достигается в на отрезке $(0,[0 ; 1])$. Проводим нормализацию $\quad$ критериев: $\quad \lambda_{1, \mathrm{~A}}\left(\mathrm{x}_{1}, \mathrm{x}_{2}\right)=\tilde{\mathrm{f}}_{1, \mathrm{~A}}\left(\mathrm{x}_{1}, \mathrm{x}_{2}\right)=\frac{\mathrm{f}_{1, \mathrm{~A}}\left(\mathrm{x}_{1}, \mathrm{x}_{2}\right)-\mathrm{f}_{1, \mathrm{~A}}^{\min }}{\mathrm{f}_{1, \mathrm{~A}}^{\max }-\mathrm{f}_{1, \mathrm{~A}}^{\min }}=$ $\frac{1000 \cdot \mathrm{x}_{2}-0}{1500-0}=\frac{2}{3} \cdot \mathrm{x}_{2}, \lambda_{2, \mathrm{~A}}\left(\mathrm{x}_{1}, \mathrm{x}_{2}\right)=\tilde{\mathrm{f}}_{2, \mathrm{~A}}\left(\mathrm{x}_{1}, \mathrm{x}_{2}\right)=\frac{\mathrm{f}_{2, \mathrm{~A}}\left(\mathrm{x}_{1}, \mathrm{x}_{2}\right)-\mathrm{f}_{2, \mathrm{~A}}^{\min }}{\mathrm{f}_{2, \mathrm{~A}}^{\max }-\mathrm{f}_{2, \mathrm{~A}}^{\min }}=\frac{0.1 \cdot \mathrm{x}_{1}-0}{0.15-0}=\frac{2}{3} \cdot \mathrm{x}_{1}$. Находим максиминное решение задачи с нормализованными критериями: $\mathrm{x}_{\mathrm{A}}^{*}=(1,1) ; \quad \tilde{\mathrm{f}}_{1, \mathrm{~A}}^{*}\left(\mathrm{x}_{1, \mathrm{~A}}^{*}, \mathrm{x}_{2, \mathrm{~A}}^{*}\right)=\frac{2}{3} ; \quad \tilde{\mathrm{f}}_{2, \mathrm{~A}}^{*}\left(\mathrm{x}_{1, \mathrm{~A}}^{*}, \mathrm{x}_{2, \mathrm{~A}}^{*}\right)=\frac{2}{3} ; \quad \mathrm{f}_{1, \mathrm{~A}}^{*}\left(\mathrm{x}_{1, \mathrm{~A}}^{*}, \mathrm{x}_{2, \mathrm{~A}}^{*}\right)=1000 ;$ $\mathrm{f}_{2, \mathrm{~A}}^{*}\left(\mathrm{x}_{1, \mathrm{~A}}^{*}, \mathrm{x}_{2, \mathrm{~A}}^{*}\right)=0.1$. Для задачи $\mathrm{A}: \mathrm{S}_{\pi, \mathrm{A}}=\left\{\mathrm{g}_{1, \mathrm{~A}}\left(\mathrm{x}_{1}, \mathrm{x}_{2}\right) \equiv \mathrm{x}_{1}+\mathrm{x}_{2} \leq 2\right.$, $\left.\mathrm{g}_{2, \mathrm{~A}}\left(\mathrm{x}_{1}, \mathrm{x}_{2}\right) \equiv \mathrm{x}_{1}-\mathrm{x}_{2} \geq-1, \mathrm{~g}_{3, \mathrm{~A}}\left(\mathrm{x}_{1}, \mathrm{x}_{2}\right) \equiv \mathrm{x}_{1}-\mathrm{x}_{2} \leq 1\right\}=\mathrm{S}_{\mathrm{a}, \mathrm{A}}-$ множество ограничений, определяющих множество Парето; $\mathrm{S}_{\mathrm{p}, \mathrm{A}}=\left\{\mathrm{g}_{4, \mathrm{~A}}\left(\mathrm{x}_{1}, \mathrm{x}_{2}\right) \equiv\right.$ $\left.\mathrm{x}_{1} \geq 0, \mathrm{~g}_{5, \mathrm{~A}}\left(\mathrm{x}_{1}, \mathrm{x}_{2}\right) \equiv \mathrm{x}_{2} \geq 0\right\}$.

Задача В. Вектор критериев $\mathrm{F}_{\mathrm{B}}=\left(\mathrm{f}_{1, \mathrm{~B}}\left(\mathrm{x}_{1}, \mathrm{x}_{2}\right), \mathrm{f}_{2, \mathrm{~B}}\left(\mathrm{x}_{1}, \mathrm{x}_{2}\right)\right)=$ $\left(1000 \cdot \mathrm{x}_{2}, 0.1 \cdot \mathrm{x}_{1}\right)=\mathrm{F}_{\mathrm{A}}, \quad$ ограничения $\mathrm{S}_{\mathrm{B}}=\left\{\mathrm{g}_{1, \mathrm{~B}}\left(\mathrm{x}_{1}, \mathrm{x}_{2}\right) \equiv \mathrm{x}_{1}+\mathrm{x}_{2} \leq 2\right.$, $\left.\mathrm{g}_{2, \mathrm{~B}}\left(\mathrm{x}_{1}, \mathrm{x}_{2}\right) \equiv \mathrm{x}_{1}-\mathrm{x}_{2} \geq-1, \mathrm{~g}_{3, \mathrm{~B}}\left(\mathrm{x}_{1}, \mathrm{x}_{2}\right) \equiv \mathrm{x}_{1} \geq 0, \mathrm{~g}_{4, \mathrm{~B}}\left(\mathrm{x}_{1}, \mathrm{x}_{2}\right) \equiv \mathrm{x}_{2} \geq 0.5\right\}$. Решаем задачу методом нормализации критериев. Последовательно находим: $\mathrm{f}_{1, \mathrm{~B}}^{\max }=1500$ (достигается $\quad$ в точке $(0.5,1.5) ; \mathrm{f}_{1, \mathrm{~B}}^{\min }=500$ (достигается в на отрезке $([0 ; 1.5], 0.5) ; \mathrm{f}_{2, \mathrm{~B}}^{\max }=0.15$ (достигается в точке $(1.5,0.5) ; \mathrm{f}_{2, \mathrm{~B}}^{\min }=0$ (достигается в на отрезке $(0,[0.5 ; 1])$. Проводим 
нормализацию $\quad$ критериев: $\quad \lambda_{1, \mathrm{~B}}\left(\mathrm{x}_{1}, \mathrm{x}_{2}\right)=\tilde{\mathrm{f}}_{1, \mathrm{~B}}\left(\mathrm{x}_{1}, \mathrm{x}_{2}\right)=\frac{\mathrm{f}_{1, \mathrm{~B}}\left(\mathrm{x}_{1}, \mathrm{x}_{2}\right)-\mathrm{f}_{1, \mathrm{~B}}^{\min }}{\mathrm{f}_{1, \mathrm{~B}}^{\max }-\mathrm{f}_{1, \mathrm{~B}}^{\min }}=$ $\frac{1000 \cdot \mathrm{x}_{2}-500}{1500-500}=\mathrm{x}_{2}-0.5, \lambda_{2, \mathrm{~B}}\left(\mathrm{x}_{1}, \mathrm{x}_{2}\right)=\tilde{\mathrm{f}}_{2, \mathrm{~B}}\left(\mathrm{x}_{1}, \mathrm{x}_{2}\right)=\frac{\mathrm{f}_{2, \mathrm{~B}}\left(\mathrm{x}_{1}, \mathrm{x}_{2}\right)-\mathrm{f}_{2, \mathrm{~B}}^{\min }}{\mathrm{f}_{2, \mathrm{~B}}^{\max }-\mathrm{f}_{2, \mathrm{~B}}^{\min }}=\frac{0.1 \cdot \mathrm{x}_{1}-0}{0.15-0}=$ $\frac{2}{3} \cdot \mathrm{x}_{1}$. Находим максиминное решение задачи с нормализованными критериями: $\mathrm{x}_{\mathrm{B}}^{*}=(0.9,1.1) ; \quad \tilde{\mathrm{f}}_{1, \mathrm{~B}}^{*}\left(\mathrm{x}_{1, \mathrm{~B}}^{*}, \mathrm{x}_{2, \mathrm{~B}}^{*}\right)=0.6 ; \quad \tilde{\mathrm{f}}_{2, \mathrm{~B}}^{*}\left(\mathrm{x}_{1, \mathrm{~B}}^{*}, \mathrm{x}_{2, \mathrm{~B}}^{*}\right)=0.6$; $\mathrm{f}_{1, \mathrm{~B}}^{*}\left(\mathrm{x}_{1, \mathrm{~B}}^{*}, \mathrm{x}_{2, \mathrm{~B}}^{*}\right)=1100 ; \mathrm{f}_{2, \mathrm{~B}}^{*}\left(\mathrm{x}_{1, \mathrm{~B}}^{*}, \mathrm{x}_{2, \mathrm{~B}}^{*}\right)=0.09$. Для задачи $\mathrm{B}: \mathrm{S}_{\pi, \mathrm{B}}=$ $\left\{\mathrm{g}_{1, \mathrm{~B}}\left(\mathrm{x}_{1}, \mathrm{x}_{2}\right) \equiv \mathrm{x}_{1}+\mathrm{x}_{2} \leq 2, \mathrm{~g}_{2, \mathrm{~B}}\left(\mathrm{x}_{1}, \mathrm{x}_{2}\right) \equiv \mathrm{x}_{1}-\mathrm{x}_{2} \geq-1, \mathrm{~g}_{4, \mathrm{~B}}\left(\mathrm{x}_{1}, \mathrm{x}_{2}\right) \equiv\right.$ $\left.\mathrm{x}_{2} \geq 0.5\right\}=\mathrm{S}_{\mathrm{a}, \mathrm{B}}-$ множество ограничений, определяющих множество Парето; $\mathrm{S}_{\mathrm{p}, \mathrm{B}}=\left\{\mathrm{g}_{3, \mathrm{~B}}\left(\mathrm{x}_{1}, \mathrm{x}_{2}\right) \equiv \mathrm{x}_{1} \geq 0\right\}$. Заметим, что множества Парето для задач А и В совпадают: $\mathrm{D}_{\pi, \mathrm{A}}=\mathrm{D}_{\pi, \mathrm{B}}$.

Замечание 1. Решение задачи $\mathrm{A}$ изменилось только за счет изменения множества пассивных ограничений задачи $\mathrm{S}_{\mathrm{p}, \mathrm{A}} \quad\left(\mathrm{S}_{\mathrm{p}, \mathrm{A}}=\right.$ $\left\{g_{4, A}\left(x_{1}, x_{2}\right) \equiv x_{1} \geq 0, g_{5, A}\left(x_{1}, x_{2}\right) \equiv x_{2} \geq 0\right\}, \quad S_{p, B}=\left\{g_{3, B}\left(x_{1}, x_{2}\right) \equiv x_{1} \geq\right.$ $0\})$.

Замечание 2. За счет изменения множества $\mathrm{S}_{\mathrm{p}, \mathrm{A}}$ произошли изменения в уступках по критериям (со значений $(33.33 \%$; $33.33 \%$ ) для задачи А, на значения (26.66\%; 40\%) для задачи В).

Здесь уступки по критериям найдены следующим образом:

- для задачи $\mathrm{A}: \quad \Delta \mathrm{f}_{1, \mathrm{~A}}=\mathrm{f}_{1, \mathrm{~A}}^{\max }-\mathrm{f}_{1, \mathrm{~A}}^{*}\left(\mathrm{x}_{1, \mathrm{~A}}^{*}, \mathrm{x}_{2, \mathrm{~A}}^{*}\right)=1500-1000=500$; относительная уступка в процентах $\Delta \mathrm{f}_{1, \mathrm{~A}} / \mathrm{f}_{1, \mathrm{~A}}^{\max } \cdot 100 \%=500 / 1500 \cdot 100 \%=33 .(3) \% ; \quad$ аналогично $\quad$ для второго критерия - $\Delta \mathrm{f}_{2, \mathrm{~A}}=\mathrm{f}_{2, \mathrm{~A}}^{\max }-\mathrm{f}_{2, \mathrm{~A}}^{*}\left(\mathrm{x}_{1, \mathrm{~A}}^{*}, \mathrm{x}_{2, \mathrm{~A}}^{*}\right)=0.15-0.1=0.05$; относительная уступка в процентах для второго критерия $\Delta \mathrm{f}_{2, \mathrm{~A}} / \mathrm{f}_{2, \mathrm{~A}}^{\max } \cdot 100 \%=0.05 / 0.15 \cdot 100 \%=33 .(3) \%$;

- для задачи $\mathrm{B}: \quad \Delta \mathrm{f}_{1, \mathrm{~B}}=\mathrm{f}_{1, \mathrm{~B}}^{\max }-\mathrm{f}_{1, \mathrm{~B}}^{*}\left(\mathrm{x}_{1, \mathrm{~B}}^{*}, \mathrm{x}_{2, \mathrm{~B}}^{*}\right)=1500-1100=400$; относительная уступка B процентах $\Delta \mathrm{f}_{1, \mathrm{~B}} / \mathrm{f}_{1, \mathrm{~B}}^{\max } \cdot 100 \%=400 / 1500 \cdot 100 \%=26 .(6) \% ; \quad$ аналогично второго критерия $\quad \Delta \mathrm{f}_{2, \mathrm{~B}}=\mathrm{f}_{2, \mathrm{~B}}^{\max }-\mathrm{f}_{2, \mathrm{~B}}^{*}\left(\mathrm{x}_{1, \mathrm{~B}}^{*}, \mathrm{x}_{2, \mathrm{~B}}^{*}\right)=0.15-0.09=0.06$; относительная уступка в процентах для второго критерия $\Delta \mathrm{f}_{2, \mathrm{~B}} / \mathrm{f}_{2, \mathrm{~B}}^{\max } \cdot 100 \%=0.06 / 0.15 \cdot 100 \%=40 \%$.

Замечание 3 . Свойство независимости решений многокритериальных задач, решаемых методом $\mathrm{M}$, от изменения множества их пассивных ограничений $S_{p}$ может быть названо $S_{p}$ инвариантностью (или $\mathrm{S}_{\mathrm{p}}$-устойчивостью) метода $\mathrm{M}$.

Замечание 4. Метод нормализации критериев (см. [1]-[4]) не является $\mathrm{S}_{\mathrm{p}}$-инвариантным. Отметим, что большинство методов, предназначенных для решения многокритериальных задач оптимизации, являются $\mathrm{S}_{\mathrm{p}}$-инвариантными. 


\section{Литература}

1. Машунин Ю.К. Методы и модели векторной оптимизации. - М.: Наука, 1986. - $140 \mathrm{c}$.

2. Кириллов Ю.В., Назимко Е.Н. Многокритериальная модель оптимизации структуры капитала// Экономический анализ: теория и практика, 2011, № 32 (239), С. 57-63.

3. Кириллов Ю.В., Назимко Е.Н. Многокритериальная задача оптимизации структуры капитала и еe решение в системе Maple// Экономика и менеджмент систем управления, 2013, т. 8, № 2.1, С. 149-160.

4. Кириллов Ю.В., Досужева Е.Е. Многокритериальная экономикоматематическая модель оценки коммерческой эффективности инвестирования// Финансовая аналитика: Проблемы и решения, 2013, № 32, C. $18-24$.

5. Список трудов [Электронный ресурс]. URL: https://sites.google.com/site/ anatolynaumov2011/home/spisok-trudov-list-of-papers (дата обращения: 23.10.2013). 